\title{
Continuous Laser Fabrication Method using Adaptive Cell Decomposition
}

\author{
Kwangho Yoon ${ }^{1}$, Kyunghan $\mathrm{Kim}^{1}$ and Jaehoon Lee ${ }^{* 1}$ \\ ${ }^{* 1}$ Dept. of High Density Energy Beam Processing \& System, KIMM, 104 Sinseongno, Yeseong-gu, Daejeon, Korea $305-343$ \\ *Email:jaholee@kimm.re.kr
}

\begin{abstract}
A one-axis on-the-fly method was developed in order to extend the processing area of the existing laser processing systems. Demands for high precision laser processing with wide areas have been increasing in a number of applications such as solar cell batteries, display parts, electronic components, the automobile industry, and so on. However, ultra-high speed laser scanner systems are limited by the size of the scanning area, for example $50 \times 50 \mathrm{~mm}$. Even though a laser scanner system that can scan a wide area has been developed recently, the distortion of the scanning quality becomes more serious as the scan area increases. In this paper, an algorithm using adaptive cell decomposition is proposed. The developed method is differentiated from the existing step and scan method in that it can guarantee continuous stage movements, which may improve the processing speed and laser fabrication quality.

DOI:10.2961/jlmn.2012.02.0017
\end{abstract}

Keywords: Laser, on-the-fly, large area, FPCB, synchronization

\section{Introduction}

Due to the increasing numbers of electrical and electronic products used in digitalization and the explosive growth in the demand for portable devices, the importance of the PCB is becoming increasingly obvious. Furthermore, the appearance of smartphones, tablet PCs, and e-paper has stimulated further demand for flexible PCBs. Because these products frequently change and are continuously updated, flexibility is required in the processing technique. Recent trends in laser micro-machining have included the shaping of ultra-precision and high speed processing of large areas, such as via-hole drilling, FPCB cutting, surface texturing, and so on $^{[1-3]}$. Using the optical system of a scanner, one method for large area processing is to scan the large area at one time using the object lens with a long focal length. However, if a scanned area of $500 \mathrm{~mm} \times 500 \mathrm{~mm}$ needs to be processed, an object lens with a focal length of 1000 $\mathrm{mm}$ is required. As well as the distortion of the lens itself, the errors due to the thermal drift of the scanner increase in proportion to the focal length. Therefore, the processing of an area of $500 \mathrm{~mm} \times 500 \mathrm{~mm}$ or more appears impossible with the scanning process using the long focus. This represents the limits of the laser for precision processing.

A hybrid method that involves the use of both a linear stage and a scanner is considered for wide area fabrication. The initial approach adopted is known as the step and scan method. A stage repeats a movement of stepping and stopping, then the scanner processes the image when the stage stops. This approach cannot accomplish continuous scanning laser processes and the precision of the image decreases at the boundaries of the area. Furthermore, it is associated with a disadvantage in that much time is required compared with continuous laser processing. Recently, an on-the-fly method was proposed to modify the step and scan method.
Scanner-stage synchronization laser processing equipment manufacturers include ESI, LPKF, and AEROTECH $^{[4-6]}$. Among these companies, Electro Scientific Industries (ESI) uses micro via-hole drilling and PCB cutting in their equipment. ESI is a micro engineering company engaged in product manufacturing and in the supply of laser systems. The scanner-stage synchronization involves unique skills when implemented as an on-the-fly algorithm. The ESI UV Laser Drilling Systems HDI5330 is capable of on-the-fly processing, and the entire stage area, measuring $533 \mathrm{~mm} \times 635 \mathrm{~mm}$, with up to $\pm 20 \mu \mathrm{m}$ accuracy guaranteed at $500 \mathrm{~mm} / \mathrm{s}$, can be processed. The German company LPKF's MicroLine 6000 model uses a PCB with an FPC applied to the cutting. The area of operation, similar to that of the ESI, is $533 \mathrm{~mm} \times 610 \mathrm{~mm}$ and the accuracy is within $\pm 20 \mu \mathrm{m}$. The company provides software known as LPKFCAM, which enables on-the-fly processing, showing a $20 \%$ reduction of the time necessary to generate the optimal path routing process. In recent years, AEROTECH in the United States has developed a scanner that synchronizes the stage on-the-fly techniques that have been introduced in laser processing. The processing section of the scanner area can move beyond the processing system. A pre-processing path optimization algorithm is introduced in order to calculate this.

Some scanners enable the synchronization of the stage. Manufacturers of these scanners, which include a control board, in Germany are RAYLASE and SCANLAB. These companies developed a control board that synchronizes the scanner and the stage, and the board is equipped with an interface module (marking on the fly; MOTF). However, in the primary applications of the developed areas of the scanner boards, such as LCD panels in large area electronic sections as an axis for laser marking on the product conveyor feed stage, and the serial number, time is limited 
regarding the use of such markers ${ }^{[7-8]}$. Many $\mathrm{FPC} / \mathrm{PCB}$ manufacturers in the United States who use the ESI process have purchased such equipment, witnessing a growing domestic demand in the development of production at an unprecedented rate.

The on-the-fly method is a control method in which the scanner and stage are synchronized. In this way, the stage moves continuously, and the accuracy of the border development is high. It can also allow an elevation of the processing quality due to the increased processing speed. In this paper, the on-the-fly method introduced uses synchronization with a scanner and a stage. The developed system delivers the stage position information to a high speed scanner board in order to develop the on-the-fly system. Also, a stage speed algorithm that uses an adaptive cell decomposition algorithm was applied ${ }^{[9]}$. The one-axis stage/two-axis scanner on-the-fly system was set up and compared with the step and scan method through various experiments.

\section{On-the-fly Systems for Continuous Laser Fabrica- tion}

\subsection{System Configuration}

Fig. 1 shows the configuration of the on-the-fly system. The on-the-fly device consists of the stage, laser scanner header, and control. The stage drives one axis. The position information is transmitted by a MOTF board and a motion board through a multi-function pulse modulation using an encoder. The control section is a motion board that controls the stage and the MOTF board, which transmits to the scanner board to calculate the position information. The scanner board consists of sections that are related to the laser and the parts that actuate the galvanometer. The laser scanner header receives information from the scanner board. The signal actuates the galvanometer through a driver. In order to use the scanner on a wide area of the stage, it is necessary to synchronize the two sections. As shown in Fig. 1, the stage position information is transmitted to the scanner. The processing information of the current position can be compared with the total position command using the stage position information. The resulting signal is passed to each galvanometer through the driver.

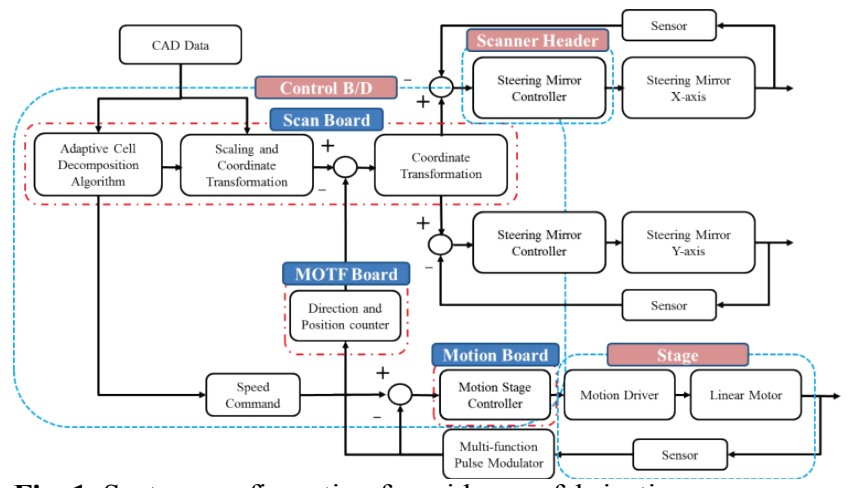

Fig. 1. System configuration for wide area fabrication.

\subsection{Cell Decomposition Algorithm}

In the next section, the speed of the stage to be synchronized is specified with regard to the scanner and stage. In order to solve this problem, a control method using the proposed synchronization algorithm was investigated. A path planning problem for a given space, which moves from the beginning to the end safely without collision, and a method to move the scanner is proposed. This requires knowledge of algorithms from the field of robotics, as well as computer graphics, artificial intelligence, games, life sciences, virtual environments, prototyping, vision surgery, CAD systems, etc. The path planning algorithms that were considered included the bug algorithm, its variants, the vector field histogram, Voronoi diagram, potential field function, probability sampling method, visibility graph method, and the method using cell decomposition algorithm. Among these, the cell decomposition algorithm was determined to be most suitable. The cell decomposition algorithm, except for the obstacles in a given space, creates empty spaces which are divided into a specific method. Then, the center of each space and its neighbors are connected to each other and a graph is configured in order to obtain the shortest path. The space was divided into $50 \mathrm{~mm}$ squares using the grid cell decomposition (GCD) algorithm because the scan area was $50 \mathrm{~mm}$. The GCD algorithm is divided into a grid of a certain size of the entire space. Then, the obstacle area, empty space, and mixed area are separated. A graph that is a GCD algorithm is constructed between the empty spaces areas. If the grid size is too small, much information in each area will require a lot of memory; otherwise, the connecting channel between the obstacles is blocked.

The processing area of the scanner used in this experiment is $50 \mathrm{~mm}$. Therefore, the cells were divided by the interval. As shown in Fig. 2, by locating the center of each cell and connecting the points, the processing path and stage movements are found. The synchronous speed is specified as follows. In Fig. 3(a) the straight line is the reference of the speed. It is assumed that the processing was $\mathrm{vf}(\mathrm{mm} / \mathrm{s})$, the processing line is $\mathrm{L}(\mathrm{mm})$, and the time is L/vf (s). Fig. 3(b) has a larger amount to process than Fig. 3(a). Thus, the stage speed must move slowly. Therefore, the following equation can be derived.

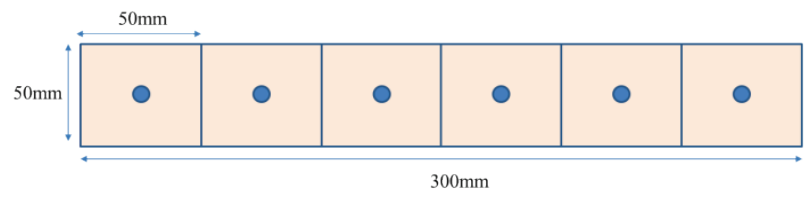

Fig. 2. Processing path using the cell decomposition.

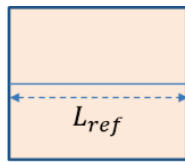

(a)

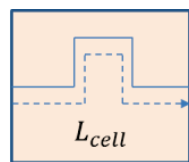

(b)

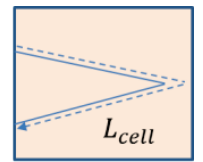

(c)
Fig. 3. Various laser processing paths divided by the cell decomposition

$$
\dot{V}_{f}=\dot{V}_{s t}+\dot{V}_{s c}
$$




$$
V_{c e l /}=\frac{L_{r e f}}{L_{c e / l}} \times V_{f},
$$

where $\dot{V}_{f}=$ velocity of the fabrication,

$\dot{V}_{s t}=$ velocity of the stage,

$\dot{V}_{S C}=$ velocity of the scanner,

$\dot{V}_{c e / /}=$ velocity per cell,

$L_{r e f}=$ velocity length per cell, and

$L_{c e / /}=$ fabrication length per cell.

\subsection{Adaptive Cell Decomposition Algorithm}

In the previous section, the stage movement was described using cell decomposition. However, if CAD has the boundary of the cell gap or scan workload differing between cells, it may cause a problem in which the processing may not be appropriate. In order to compensate this problem, the workload per cell was compared and an algorithm for splitting or merging is proposed. Fig. 4 presents the algorithm tree of the proposed algorithm. $\mathrm{C} 1$ is first divided cell, and the cell numbers follow accordingly. C1 and $\mathrm{C} 2$ cell is compared to the workload. $k$ is the ratio of the workload between each cell. In this paper, the ratio of $k$ was defined as follows.

$$
\begin{aligned}
& \frac{C_{m+1}}{C_{m n}}=k_{n} \\
& \frac{C_{m+2}}{C_{m+1}}=k_{n+1} \\
& \frac{C_{m+3}}{C_{m+2}}=k_{n+2} \\
& \frac{C_{m+4}}{C_{m+3}}=k_{n+3}
\end{aligned}
$$

Where, $c$ is a divided cell. $w$ is a workload. $n$ is a number. And the scope of workload was defined as follows.

$$
\begin{aligned}
& w_{n}: k=1 \\
& w_{n+1}: 1<k \leq \frac{1}{2} \text { or } 1<k \leq 2 \\
& w_{n+2}: \frac{1}{2}<k \leq \frac{1}{4} \text { or } 2<k \leq 4 \\
& w_{n+3}: \frac{1}{4}<k \leq \frac{1}{8} \text { or } 4<k \leq 8
\end{aligned}
$$

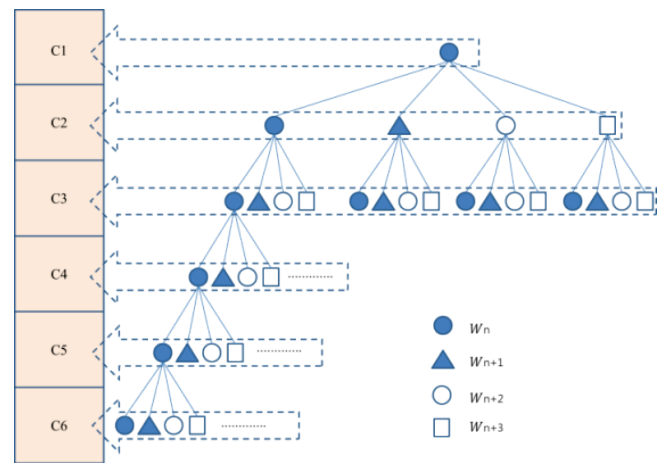

Fig. 4. Algorithm tree according to the scan workloads of cells.
Fig. 5 presents an example of the adaptive cell decomposition method. By comparing the scan workload of $\mathrm{Cl}$ and $\mathrm{C} 2$, the cells are divided according to the ratio. This is repeated for the remaining cells. Then, moving backwards from the last section of the divided cells, the values are added to the previous section.

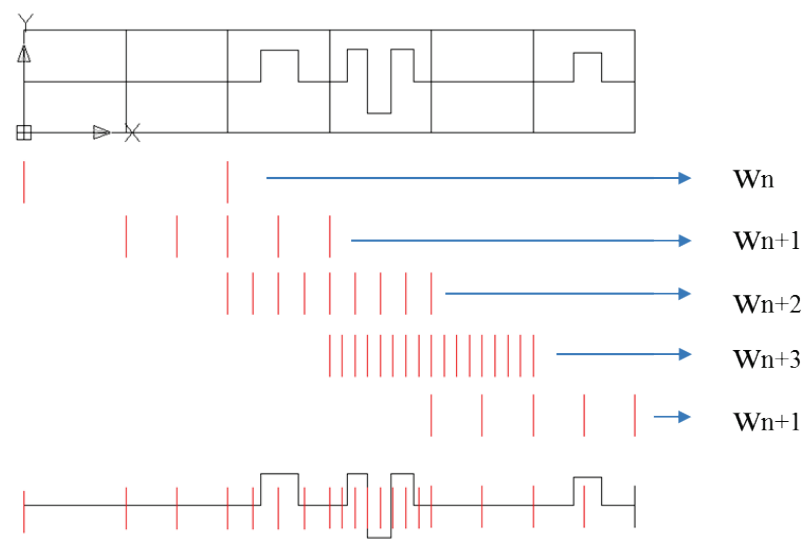

Fig. 5. Cell merger using adaptive cell decomposition.

\section{Experiment}

\subsection{Comparison of Step and Scan Method with the On-the-fly Method}

A scanner control board that can accept two-axis stage signals is still under development; thus, only a one-axis stage/two-axis scanner on-the-fly system is developed and tested in the current research, as shown in Fig. 5. An IPG fiber laser with a $30 \mathrm{~ns}$ pulse width, $12 \mathrm{~W}$ power, and 1064 $\mathrm{nm}$ wavelength was used for the on-the-fly marking experiment. A CTI scanner head with an aperture of $10 \mathrm{~mm}$ and a focal length of $100 \mathrm{~mm}$ was also used. The stage of the DASAROBOT has a $400 \mathrm{~mm}$ stroke with a maximum speed of $500 \mathrm{~mm} / \mathrm{s}$ and a precision of approximately $5 \mathrm{um}$. For a low pulse repetition rate of $1 \mathrm{kHz}$, the marking precision between the step and scan method and the on-the-fly method is compared. The marking speed is $300 \mathrm{~mm} / \mathrm{s}$, and the scanner working area is $50 \mathrm{~mm} \times 50 \mathrm{~mm}$. In the step and scan method, the stage is motionless while the scanner working. After the scanner operates within the working area, the stage shifts $50 \mathrm{~mm}$ to the right to begin with the next scanning process. The step and scan movement processes are repeated in order to mark the entire area. However, for the on-the-fly system, the stage and scanner move and work together.

Comparisons of the spots marked by the two methods are shown in Fig. 6. In the scanner working area of both methods, the distances between the marking spots are almost constant in all directions. In the step and scan method, irregular spots are observed at the boundary of the scanner working area. This is because the exact marking positions cannot be controlled due to the irregular start and stop movements of the stage. However, the on-the-fly method shows an almost consistent marking distance between the spots in the boundary region and the working area. This is a critical advantage of the on-the-fly system over the step and scan method. Fig. 6 shows the results of the laser marking pattern from the two methods. In the red rectan- 
gles, it can be seen that the laser pulse was processed too close together twice. Fabricated plate is anodized aluminum. In the future, it will be applied to the FPCB.
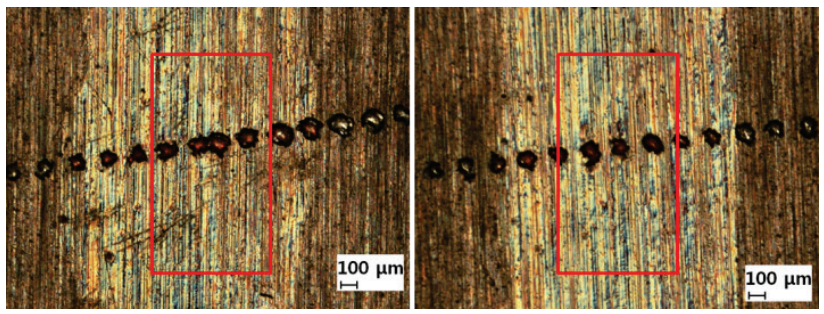

Fig. 6. Results of the laser marking pattern from the two methods: the step \& scan method (left) and the on-the-fly method (right).

\subsection{Precision Test of the On-the-fly Method}

A ruler was used to measure the accuracy of the developed system. A straight line of $130 \mathrm{~mm}$ in length was tested on the ruler using laser fabrication. In order to measure the precise length of fabrication result we used the stage with a vision. The stage has a maximum speed of $1.2 \mathrm{~m} / \mathrm{s}$ and maximum acceleration of $15 \mathrm{~m} / \mathrm{s}^{2}$, Accuracy is $\pm 1.8 \mu \mathrm{m}$, Repeatability is $\pm 0.24 \mu \mathrm{m}$, Straightness is $2.4 \mu \mathrm{m}$, Flatness of the linear motor is $3.8 \mu \mathrm{m}$. The camera used XCL-5005 models of the sony, the lens used MML4-HR65 models of the moritex. FOV(field of view) is $2.2 \mathrm{~mm}$, WD(working distance) is $65 \mathrm{~mm}$. As shown in Fig. 7, A is a start point. B is an end point that the stage is moved $130 \mathrm{~mm}$. $\mathrm{C}$ is a real end point which have finished the laser processing. The error is approximately $36 \mathrm{um}$.

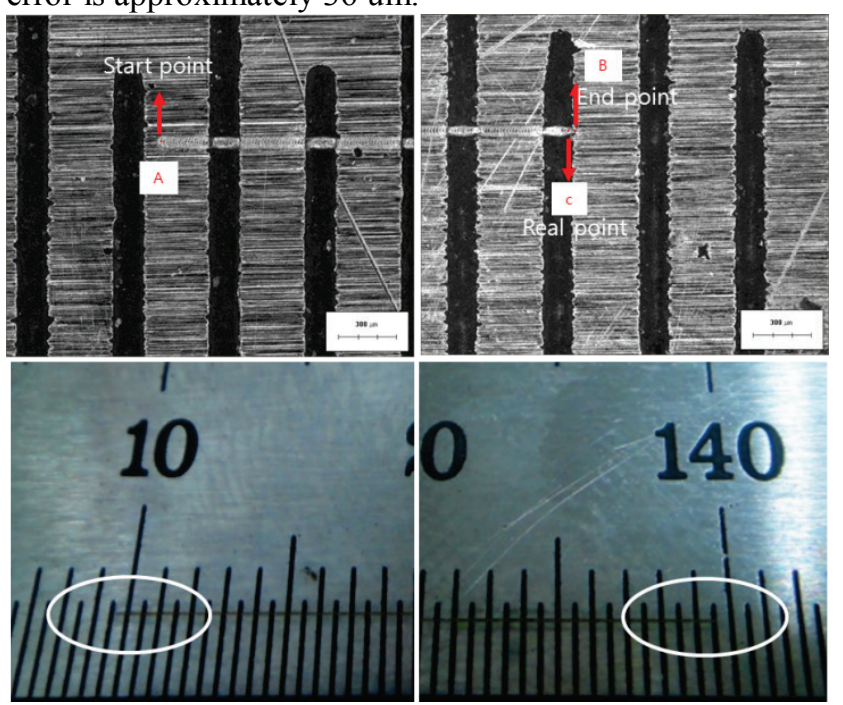

Fig. 7. Precision test of the on-the-fly method using a ruler to measure the distances.

\subsection{PCB Pattern Test using the Adaptive Cell De-} composition Algorithm

Using the proposed algorithm, the CAD file was tested using the FPCB pattern as a continuous process. Fig. 8 shows the CAD dimensions $(\mathrm{mm})$ and the fabricated sheet result. When processing this pattern, the galvanometer and stage signals are as shown in Fig. 9. In Fig. 9, it can be seen that the scan area did not exceed $50 \mathrm{~mm}$. This indicates that the scan area is not exceeded during the laser fabrication time. Furthermore, this demonstrates that it can obtain the correct process.
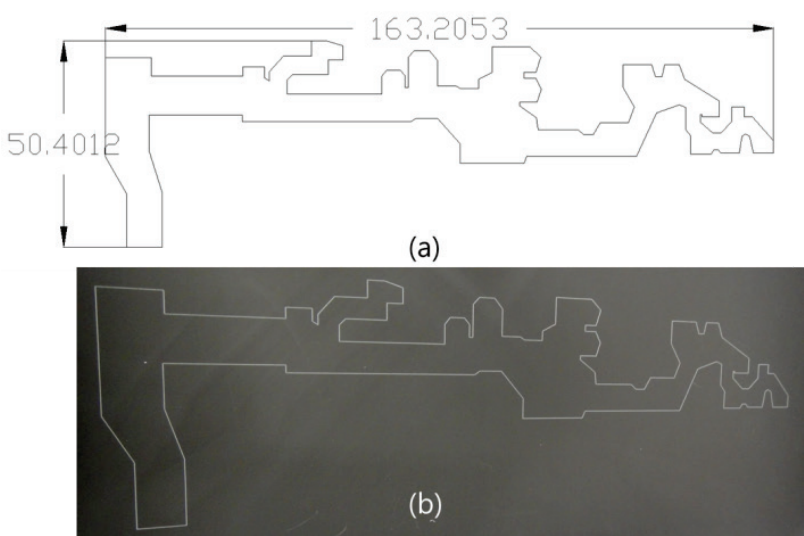

Fig. 8. (a) FPCB pattern CAD data (b) FPCB pattern fabrication result.

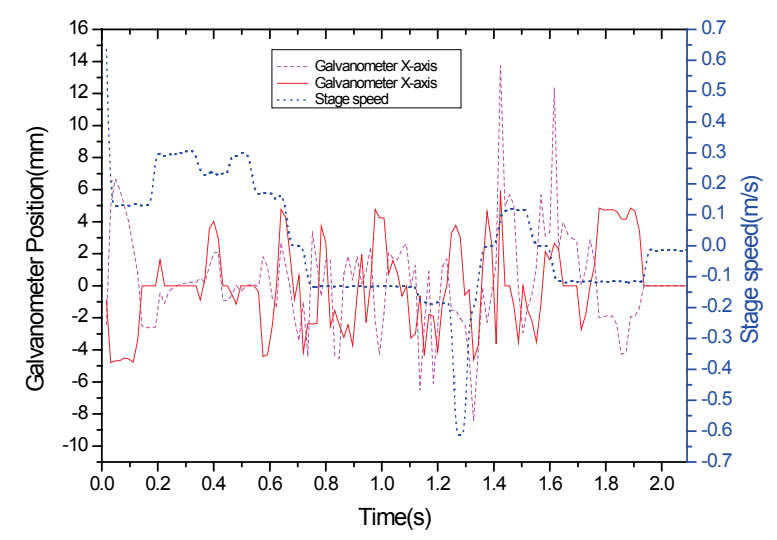

Fig. 9. Signals for the FPCB pattern fabrication.

\section{Conclusion}

A one-axis stage with a synchronized scanner system was developed. Also, the scan and MOTF board required to synchronize the movement was developed. Furthermore, the position information could be delivered to the scan board. In addition, the stage was synchronized with the scanner by the proposed method using adaptive cell decomposition.

In the one-axis stage/two-axis scanner on-the-fly system, the marking distances between the spots were measured. A constant distance was measured at both the scanner working areas and the boundary regions. However, in the step and scan method, irregular marking points were observed, which may lead to poor quality fabrications.

\section{Acknowledgments and Appendixes}

This work was supported by the Korean Ministry of Knowledge Economy within the project "Precision/High Speed Laser Cutting System Core Technology Development" (Industrial Source Technology Development, Project Number: 10033829).

\section{References}

[1] S. Nikumb, Q. Chen, C. Li, H. Reshef, H. Y. Zheng, H. Qiu, and D. Low, "Precision glass machining, drilling 
and profile cutting by short pulse lasers", Thin Solid Films, Vol. 477, pp. 216-221, 2007.

[2] D. Shin, J. Lee, H. Sohn, J. Noh, and B. Paik, "A FPCB cutting process using a pico-second laser," JLMN, Vol. 5,. No. 1, pp. 48-52 (2010).

[3] H. J. Kang, H. J. Kim, J. S. Kim, W. Y. Choi, W. S. Chu, and S. H. Ahn, "Laser marking system for light guide panel using design of experiment and web-based prototyping," Robotics and Computer-Integrated Manufacturing, (In press) .

[4] http:// www.esi.com

[5] http:// www.aerotech.com

[6] http:// www.lpkf.com

[7] T. Kim, S. Park, H. Oh, and Y. Shin, "Analysis of the laser patterning inside light guide panel", Optics \& Laser Technology 39, pp. 1437-1442, 2007.

[8] T. Borangiu, A. Dogar, and A. Dumitrache, "Constraints-based Motion Planning for an Automatic, Flexible Laser Scanning Robotized Platform," Automation, Quality and Testing, Robotics, Vol. 2, pp. 6569, 2008.

[9] F. Lingelbach, "Path planning using probabilistic cell decomposition," in Proc. of the IEEE Int. Conf. on Robotics and Automation, pp. 467-472(2004)

(Received: June 07, 2011, Accepted: March 15, 2012) 